\title{
Análisis Morfológico y Funcional del Músculo Pterigoideo Lateral: Una Revisión de la Literatura
}

\author{
Morphological and Functional Analysis of the Lateral \\ Pterygoid Muscle: A Review of the Literature
}

\author{
Constanza Farfán ${ }^{1,2}$; Jonathan Roig ${ }^{3}$; Bryan Quidel $^{3}$ \& Ramón Fuentes $^{1}$
}

FARFÁN, C.; ROIG, J.; QUIDEL, B. \& FUENTES, R. Análisis morfológico y funcional del músculo pterigoideo lateral: Una revisión de la literatura. Int. J. Morphol., 38(6):1713-1721, 2020.

RESUMEN: El músculo pterigoideo lateral (MPL) es una estructura compleja y variable, poder determinar su anatomía exacta, relaciones vecinas, origen e inserciones, ayuda a los clínicos a comprender de mejor forma su función en el sistema estomatognático. En esta revisión se busca analizar la literatura que ayude a esclarecer la función antagónica de las cabezas del músculo pterigoideo lateral, desde un punto de vista nervioso, la descripción de su origen e inserciones y sus posibles variaciones anatómicas, además del análisis de sus funciones evaluado a través de la literatura tradicional y compararlo con lo descrito en artículos originales. Se analizaron diferentes bases de datos electrónicos y libros, con criterios de inclusión e exclusión claramente definidos, la lectura fue llevada a cabo por dos investigadores de manera independiente consultando de ser necesario con un tercer investigador. Esta revisión incluyo un total de 11 artículos y 4 libros atingentes a nuestro tema de estudio. Se expusieron los resultados a través de tablas de extracción de datos, que incluyó las funciones, inervación, el origen e inserción y las variaciones anatómicas del MPL. Nuestros resultados muestran que se identificaron claramente los elementos comunes de origen del MPL, pero su inserción mostró variaciones entre los distintos estudios, tanto en el porcentaje de fibras unidas como a los elementos anatómicos insertados. Considerando las funciones antagónicas de sus dos cabezas, autores lo han descrito como dos músculos diferentes, sin embargo desde un punto de vista nervioso, esta teoría no es apoyada. Finalmente comprender las funciones del MPL durante su acción es complejo, ya que la gran mayoría de los estudios disponibles utilizan cadáveres o electromiografía por lo que creemos que el desarrollo de metodologías menos invasivas y dolorosas, ayudarían a comprender el comportamiento de este músculo durante su función y como las variaciones anatómicas influyen en estas.

PALABRAS CLAVE: Músculo Pterigoideo Lateral; Anatomía funcional; Variaciones anatómicas; Articulación Temporomandibular.

\section{INTRODUCCIÓN}

El músculo pterigoideo lateral (MPL), ha sido interés de investigación durante los últimos años. Poder determinar su anatomía exacta, relaciones vecinas, origen e inserciones, ayuda a los clínicos a comprender de mejor forma su función en el sistema estomatognático (Gutiérrez \& Grossman, 2010). Para determinar su morfología se ha recurrido a distintas técnicas, desde las tradicionales como una disección (Akar et al., 2009), hasta la utilización de herramientas imagenológicas (Bernal-Mañas et al., 2016). Se ha descrito en la literatura como un músculo de dos cabezas, cónico, corto, grueso, triangular e irregular, constituido por capas de músculo aponeurótico que le confieren una estructura peniforme, todas estas definiciones han sido muy dis- cutidas por la comunidad científica (Testut \& Latarjet 1989; Mazza et al., 2009; Norton, 2007; Kendall et al., 2005; El Haddioui et al., 2005).

En cuanto a la morfología del MPL, se ha descrito que está compuesto por dos cabezas una superior o esfenoidal (MPL-S) y otra inferior o pterigoidea (MPL-I), que presentan distinta disposición de sus fibras, horizontales en la parte superior y oblicuas ascendentes en la inferior (Velayos \& Santana, 1998; Matsunaga et al., 2009). Ambas cabezas difieren tanto en su origen e inserción, otorgándole a este, una amplia gama de movimientos (Usui et al., 2008). La cabeza superior se origina en la superficie

\footnotetext{
${ }^{1}$ Dental School, Research Centre for Dental Sciences (CICO), Universidad de La Frontera, Temuco, Chile.

${ }^{2}$ Universidad Adventista de Chile, Chillán, Chile.

${ }^{3}$ Undergraduate student, Dental School, Universidad de La Frontera, Temuco, Chile.
} 
lateral del ala mayor del esfenoides y cresta infratemporal (Langton \& Eggleton, 1992; Kendall et al.), mientras que su inserción ha sido muy discutida y estudiada durante los últimos años, describiéndose que estaría dividida entre el cóndilo de la mandíbula (CM), la cápsula articular (CA) y el disco articular (DA) (Naidoo \& Juniper, 1997). Antonopoulou et al. (2013) describen tres formas distintas de inserción del MPL-S, siendo la más frecuente, a nivel del proceso condilar, en la fóvea pterigoidea y DA (55\%), seguida por la inserción en el CM $(27,8 \%)$ y el $16,7 \%$ se insertó a nivel del complejo cápsula-disco articular. En cuanto al porcentaje de fibras que se insertan en el disco articular se ha establecido mediante técnicas histológicas y resonancia magnética principalmente, los porcentajes que varían de un 29,5\% (Naidoo \& Juniper), a un 69,3\% de inserción del MPL-S (Pompei Filho et al., 2010). A diferencia de la cabeza superior, el MPL-I, no tiene mayor discusión en la literatura, originándose en la cara lateral de la lámina lateral del proceso pterigoides para insertarse en la fosita pterigoidea en el cuello del proceso condilar de la mandíbula (Norton; Alves \& Candido, 2009).

La función de este músculo, va a depender de la cabeza que actúe. El MPL-S ejerce su acción durante el cierre mandibular (Lopes, 2004) en acciones como masticar (Biasotto, 2005), rechinar de dientes y en la deglución (Velayos \& Santana, 1998). La fijación miofascial ejercida por estas fibras impide la invaginación de la caápsula articular (CA) durante el movimiento hacia posterior del cóndilo y disco articular (Langton \& Eggleton). El MPL-I, ejerce su acción durante la apertura y protrusión mandibular

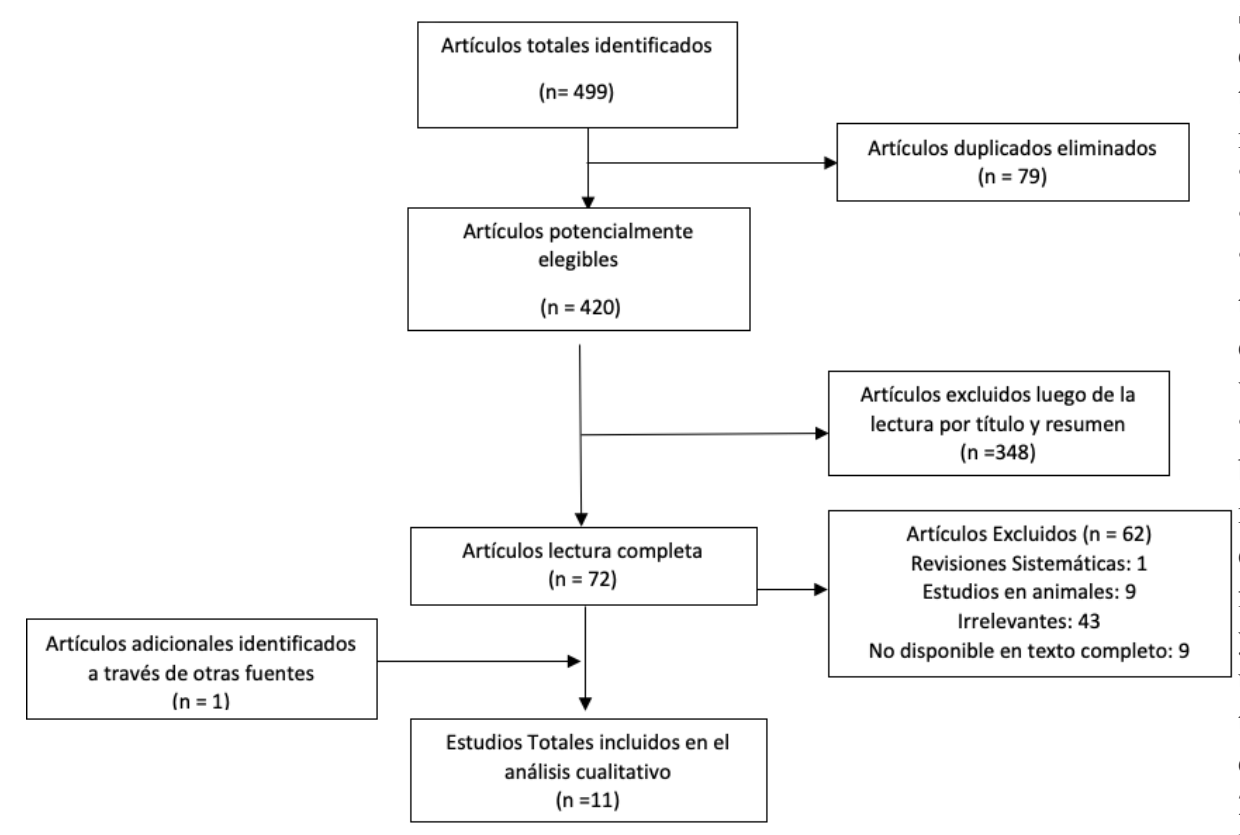

Fig. Diagrama de Flujo, búsqueda sistemática.
(Velayos \& Santana, 1998; Lopes), esta última con la intervención de los músculos elevadores de la mandíbula. La función que ejercen ambas cabezas actuando en conjunto, consiste en tirar hacia adelante el CM, el DA y la CA en dirección hacia el tubérculo articular, función en la que no puede ser sustituido por ningún otro músculo (Velayos \& Santana, 1998). Se ha argumentado que las dos cabezas del MPL, son recíprocamente activas durante el ciclo masticatorio. Sin embargo, basado en aspectos anatómicos, biomecánicos, y electromiográficos, autores sugieren que las dos cabezas se consideren músculos independientes (Ferrario \& Sforza, 1996; Aziz et al., 1998). Sin embargo, han surgido preguntas sobre la precisión de estos datos, planteándose la hipótesis de que la partición no puede completarse sin un suministro nervioso independiente.

De acuerdo a lo anterior, en esta revisión se busca analizar la literatura que ayude a esclarecer la función antagónica de las cabezas del músculo pterigoideo lateral, desde un punto de vista nervioso, la descripción de su origen e inserciones y sus posibles variaciones anatómicas, además del análisis de sus funciones evaluado a través de libros la literatura tradicional.

\section{MATERIAL Y MÉTODO}

Se realizó una revisión de la literatura con búsqueda sistemática, utilizando los criterios PRISMA. Las bases de datos electrónicas empleadas fueron PUBMED, SCOPUS, EMBASE, WEB OF SCIENCE SciELO y LILACS. Los términos de búsqueda utilizados fueron "lateral pterygoid muscle", "pterygoid muscles", "magnetic resonance", "dissection", "fibers", "insertion". "origin", "function", "anatomy", "irrigation" y "innervation". Estos términos fueron utilizados individualmente y en combinación a través de los operadores booleanos "OR" y "AND" en los diferentes buscadores, con el empleo de términos MeSH. La búsqueda dentro de las bases de datos electrónicas fue realizada entre diciembre 2019 y enero 2020. Además, se realizó una búsqueda manual de la literatura donde se revisaron 19 libros del área, con años entre 1989 y 2015 de los cuales consideramos 4 libros, según nuestro objetivo. 
Criterios de elegibilidad. Esta revisión incluyo artículos científicos publicados en inglés, español y portugués, sin un límite de tiempo atingentes a nuestro objetivo de estudio, que identificaron el MPL en humanos vivos y cadáveres, a través de técnicas de disección e imagenológicas, describiendo la morfología, funcionalidad y relaciones anatómicas. Se excluyeron artículos científicos y tesis de diseño experimental o cuasi experimental que se realizaron en animales y revisiones sistemáticas.

Selección de datos y extracción de artículos. La selección de los artículos fue realizada por dos investigadores de forma independiente; analizando en primera instancia Título y Resumen de los diferentes estudios, los artículos que cumplían con los criterios de elegibilidad se analizaron en texto completo para confirmar su relevancia. Cuando hubo controversia y diferencias en la aplicación de los criterios de selección, un tercer investigador realizó el análisis y determinación final para la incorporación o exclusión del estudio.

Estrategia de búsqueda: La búsqueda en las diferentes bases de datos, arrojó un total de 499 artículos relacionados con los términos buscados. Los artículos duplicados, excluidos y seleccionados se detallan en el diagrama de flujo de la (Fig. 1). Debido a la temática que se deseó investigar, fue necesario realizar una búsqueda manual, en libros relacionados al tema, publicados en idiomas español, inglés y portugués (Fig. 2).

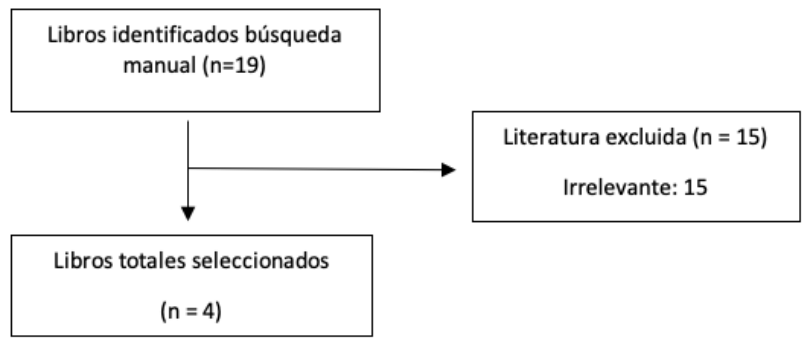

Fig. Diagrama de Flujo, búsqueda manual.

\section{RESULTADOS}

De la búsqueda sistemática se encontraron 499 artículos dentro de las siguientes bases de datos electrónicas: PUBMED, LILACS, SciELO, SCOPUS, WEB OF SCIENCE y EMBASE. De los cuales 79 artículos fueron eliminados ya que se encontraban duplicados, luego se realizó una revisión de Título y Resumen dejando los artículos que aportaban información a la revisión según los parámetros descritos en los criterios de selección. Una vez finalizada la primera revisión, fueron seleccionados un total de 73 artículos, que se revisaron a texto completo, luego de esta lectura se excluyeron 62 artículos: Una revisión sistemática, 9 artículos eran estudios en animales, 43 artículos fueron considerados irrelevantes para nuestro obje-

Tabla I. Inervación del músculo pterigoideo lateral.

\begin{tabular}{|c|c|c|c|c|c|}
\hline Referencia & Muestra & Metodología & $\begin{array}{l}\text { Resultados Inervación } \\
\text { MPL }\end{array}$ & Cabeza superior & Cabeza inferior \\
\hline $\begin{array}{l}\text { Akita et al., } \\
2000\end{array}$ & $\begin{array}{l}14 \text { cadáveres } \\
\text { japoneses ( } 8 \\
\text { hombres y } 6 \\
\text { mujeres) }\end{array}$ & Disección & $\begin{array}{l}\text { Todas las muestras fueron } \\
\text { inervadas por ramos del nervio } \\
\text { temporal profundo ant erior. }\end{array}$ & $\begin{array}{l}\text { El } 61,5 \% \text { de las muestras, } \\
\text { fueron inervadas por ramas } \\
\text { del nervio temporal } \\
\text { profundo medio. }\end{array}$ & $\begin{array}{l}\text { El 76,9\% de las muestras, } \\
\text { fueron iner vadas por el tronco } \\
\text { del Nervio mandibular. }\end{array}$ \\
\hline $\begin{array}{l}\text { Aziz et al., } \\
1998\end{array}$ & $\begin{array}{l}20 \text { cadáveres } \\
\text { Afroamerican } \\
\text { os y } \\
\text { caucásicos ( } 13 \\
\text { hombres y } 7 \\
\text { mujeres). }\end{array}$ & Disección & $\begin{array}{l}\text { El } 75 \% \text { de las muestras la } \\
\text { inervación de ambas cabezas } \\
\text { se originó en el nervio } \\
\text { mandibular o nervio bucal. } \\
\text { En el } 20 \% \text { de las muestras las } \\
\text { cabezas recibían inervación } \\
\text { independiente del nervio } \\
\text { temporal anterior, bucal y/o } \\
\text { mandibular. }\end{array}$ & $\begin{array}{l}\text { El patrón nervioso más } \\
\text { prevalente, fueron ramas } \\
\text { nerviosas independientes } \\
\text { adicionales origin adas del } \\
\text { nervio temporal profundo, } \\
\text { bucal o mandibular. }\end{array}$ & $\begin{array}{l}\text { El patrón nervioso más } \\
\text { prevalente, fueron ramas } \\
\text { nerviosas adicionales } \\
\text { independientes originadas del } \\
\text { nervio bucal o mandibular. }\end{array}$ \\
\hline $\begin{array}{l}\text { Kim et al., } \\
2003\end{array}$ & $\begin{array}{l}24 \text { cadáveres } \\
\text { coreanos }(16 \\
\text { hombres y } 8 \\
\text { mujeres) }\end{array}$ & Disección & $\begin{array}{l}\text { Todos los elementos nerviosos } \\
\text { se originaron del nervio } \\
\text { mandibular. }\end{array}$ & $\begin{array}{l}\text { En el } 45.8 \% \text { de las } \\
\text { muestras los ramos } \\
\text { nerviosos se originaban del } \\
\text { nervio bucal. }\end{array}$ & $\begin{array}{l}\text { En el } 58.3 \% \text { de las muestras la } \\
\text { inervación se origi nó del nervio } \\
\text { bucal y el tronco nervioso } \\
\text { mandibul ar. }\end{array}$ \\
\hline $\begin{array}{l}\text { Akita et al., } \\
2001\end{array}$ & $\begin{array}{l}34 \text { cadáveres } \\
\text { japoneses } \\
\text { adultos: } 18 \\
\text { hombres y } 16 \\
\text { mujeres }\end{array}$ & Disección & $\begin{array}{l}\text { Ramas del nervio temporal } \\
\text { profundo ant erior. }\end{array}$ & $\begin{array}{l}\text { En el } 65,2 \% \text { de las } \\
\text { muestras: ramas del nervio } \\
\text { temporal profundo medio. }\end{array}$ & $\begin{array}{l}\text { En el } 72.7 \% \text { de las muestras el } \\
\text { tronco prin cipal del nervio } \\
\text { mandibul ar a través de su } \\
\text { trayecto en la superficie medial. }\end{array}$ \\
\hline
\end{tabular}


tivo de estudio y 9 artículos no estaban disponible en fulltext, quedando 10 artículos seleccionados de la revisión y la aplicación de los criterios, a esto se le agrega un artículo identificado de otras fuentes que cumple con nuestro objetivo de estudio, quedando un total de 11 artículos seleccionados que evaluaron la inervación de las cabeza superior e inferior del MPL (Tabla I), el origen y la inserción del músculo pterigoideo lateral (Tabla II) y las variaciones anatómicas reportadas (Tabla III).

Tabla II. Origen e inserción del músculo pterigoideo lateral.

\begin{tabular}{|c|c|c|c|c|c|}
\hline \multirow[t]{2}{*}{ Referencia } & \multirow[t]{2}{*}{ Muestra } & \multirow[t]{2}{*}{ Origen } & \multirow[t]{2}{*}{ Metodología } & \multicolumn{2}{|c|}{ Resultados } \\
\hline & & & & Origen & Inserción \\
\hline $\begin{array}{l}\text { Matsunaga et } \\
\text { al., } 2009\end{array}$ & $\begin{array}{l}10 \text { hombres } \\
\text { y } 1 \text { o } \\
\text { mujeres. } \\
\text { (promedio } \\
79,6 \text { años). }\end{array}$ & Japón & Disección & $\begin{array}{l}\text { MPL: Fibras longitudinales: } \\
\text { superficie lateral de la placa } \\
\text { pterigoidea lateral. } \\
\text { Fibras horizontales: cresta } \\
\text { infratemporal y la superficie } \\
\text { inferior del ala mayor del } \\
\text { esfenoides. } \\
\text { MPL-S---- } \\
\text { MPL-I ---- }\end{array}$ & $\begin{array}{l}\text { MPL: mitad medial de la superficie } \\
\text { anterior de la fóvea pt erigoidea y la } \\
\text { superficie medial del proc eso condilar. } \\
\text { MPL-S: En su mayoría en el CM. } \\
\text { Fibras horizontales superficiales: } \\
\text { superficie inferior del DA } \\
\text { No existe un límite entre la inserción de } \\
\text { las fibras musculares horizontales y } \\
\text { longitudinales. } \\
\text { MPL-I: --- }\end{array}$ \\
\hline $\begin{array}{l}\text { Antonopoulou } \\
\text { et al., } 2012\end{array}$ & $\begin{array}{l}8 \text { ho mbres y } \\
10 \text { mujeres } \\
\text { (promedio } \\
76,9 \text { años). }\end{array}$ & Grecia & Disección & $\begin{array}{l}\text { MPL: --- } \\
\text { MPL-S: Ala mayor del esfenoides } \\
\text { y cresta infratemporal. } \\
\text { MPL-I: Placa pterigoidea lateral. } \\
\text { Cabeza media: Superficie del ala } \\
\text { mayor del esfenoides. }\end{array}$ & $\begin{array}{l}\text { MPL: --- } \\
\text { MPL-S: El } 55.5 \% \text { se inserta en el CM y } \\
\text { DA. } \\
\text { El } 27.8 \% \text { se inserta sólo en el CM. } \\
\text { El } 16.7 \% \text { sólo se ins erta en el DA. } \\
\text { MPL-I: Fóvea pterigoidea. } \\
\text { Cabeza media: ---- }\end{array}$ \\
\hline $\begin{array}{l}\text { Siéssere et al., } \\
2008\end{array}$ & $\begin{array}{l}9 \text { hombres } \\
\text { (promedio } \\
40 \text { años). }\end{array}$ & Brasil & Disección & $\begin{array}{l}\text { MPL: --- } \\
\text { MPL-S: Ala esfenoidal. } \\
\text { MPL-I: Lamina lateral del } \\
\text { proceso pterigoi deo. }\end{array}$ & $\begin{array}{l}\text { MPL: --- } \\
\text { MPL-S: DA. } \\
\text { MPL-I: Fosa pterigoidea. }\end{array}$ \\
\hline $\begin{array}{l}\text { Haddioui } \\
\text { al., } 2005\end{array}$ & $\begin{array}{l}84 \text { hombres } \\
\text { y } \\
95 \text { mujeres } \\
\text { (promedio } \\
\text { de } 73 \text { años). }\end{array}$ & $\begin{array}{l}\text { No } \\
\text { identificado }\end{array}$ & $\begin{array}{l}\text { Disección } \\
\text { Imagenología }\end{array}$ & $\begin{array}{l}\text { MPL: Cara infratemporal del ala } \\
\text { mayor del esfenoides y los } 2 / 3 \\
\text { inferiores de la cara lateral de la } \\
\text { lámina lateral del proceso } \\
\text { pterigoides del hueso esfenoides. } \\
\text { MPL-S: --- } \\
\text { MPL-I: --- } \\
\text { BLE EN EL ESTUDIO. }\end{array}$ & $\begin{array}{l}\text { MPL: DA a nivel de su mitad medial y } \\
\text { cuello del CM con una inserción } \\
\text { triangular de base superior. } \\
\text { MPL-S: --- } \\
\text { MPL-I: --- }\end{array}$ \\
\hline
\end{tabular}

\section{DISCUSIÓN}

La descripción de las funciones del MPL, son motivo de investigación constante, se ha descrito como una unidad única, conformada por dos cabezas con funciones específicas (Carpentier et al., 1988; Antonopoulou et al.). En la literatura revisada, pudimos percatarnos que al momento de describir las funciones del MPL, los autores se basaban en su gran mayoría, en la literatura tradicional citando libros en sus definiciones, mientras que los estudios intentaron evaluar sus funciones ha sido en su mayoría a través de electromiografía, pero dadas las características de la ubica-
En cuanto a la búsqueda manual, un total de cuatro libros fueron analizados, tres correspondientes a anatomía aplicada y uno a especialidades odontológicas; tres libros fueron publicados en idioma español y uno en portugués. Los libros seleccionados, fueron aquellos que mencionaban las funciones del músculo pterigoideo lateral, ya sea de forma conjunta (ambas cabezas) o que incluían un análisis de función de acuerdo a cada cabeza (Tabla IV). Se decidió revisar en libros este objetivo, ya que los artículos revisados, se basan en libros para la descripción de las funciones del MPL. 
Tabla III. Variaciones anatómicas músculo pterigoideo lateral.

\begin{tabular}{|c|c|c|c|c|}
\hline Referencia & Muestra & Origen & Metodología & Resultado \\
\hline $\begin{array}{l}\text { Pompei et al., } \\
2009\end{array}$ & $\begin{array}{l}178 \text { A TM de } \\
\text { individuos adultos } \\
\text { sanos. } \\
(20-45 \text { años }) .\end{array}$ & $\begin{array}{l}\text { No } \\
\text { especifica }\end{array}$ & Imagenología & $\begin{array}{l}\text { Observa una tercera cabeza del MPL. } \\
\text { La prevalencia de la tercera cabeza del MPL fue del } \\
20,22 \% \text {. La inserción se realizó completamente en } \\
\text { el disco de la ATM, el área promedio de inserción } \\
\text { fue de } 4,14 \pm 1,35 \mathrm{~mm} \text { en mujeres y } 4,67 \pm 2,68 \\
\text { mm en hombres, sin diferencias significativas. }\end{array}$ \\
\hline $\begin{array}{l}\text { Akar et al., } \\
2009\end{array}$ & $\begin{array}{l}25 \text { cadáveres de } \\
\text { hombres adultos. }\end{array}$ & $\begin{array}{l}\text { Región } \\
\text { del Egeo }\end{array}$ & Disección & $\begin{array}{l}\text { Observa una tercera cabeza del MPL. } \\
\text { Se encontró que el MPL se originó con } 3 \text { cabezas: } \\
\text { Superior, inferior, y "cabeza interna” siendo la } \\
\text { inferior } 3 \text { veces más ancha que la cabeza superior. }\end{array}$ \\
\hline $\begin{array}{l}\text { Antonopoulo } \\
\text { u et al., } 2012\end{array}$ & $\begin{array}{l}8 \mathrm{~h} \text { ombres y } 10 \\
\text { mujeres } \\
\text { (promedio de } 79,6 \\
\text { años). }\end{array}$ & Grecia & Disección & $\begin{array}{l}\text { Observa una tercera cabeza del MPL. } \\
\text { Se identificó la presencia de una cabeza media en el } \\
22.2 \% \text { de los casos y se originaba en el ala mayor } \\
\text { del hueso esfenoides. }\end{array}$ \\
\hline $\begin{array}{l}\text { Akita et al., } \\
2001\end{array}$ & $\begin{array}{l}18 \text { hombres y } 16 \\
\text { mujeres, adultos. }\end{array}$ & Japón & Disección & $\begin{array}{l}\text { Observa un haz muscular anómalo entre el Músculo } \\
\text { Temporal (MT) y MPL ( } 4,5 \% \text { de las muestras). Se } \\
\text { originaba de la superficie media de la porción } \\
\text { antero-medial del MT y se extendía hasta la } \\
\text { superficie ínfero -lateral del MPL-I. } \\
\text { En una muestra el origen también se dio en la cresta } \\
\text { infratemporal del ala mayor del esfenoides. } \\
\text { Es considerado como el músculo "pterygoideus } \\
\text { proprius". }\end{array}$ \\
\hline $\begin{array}{l}\text { Snoeck et al., } \\
2010\end{array}$ & $\begin{array}{l}21 \text { hombres y } 18 \\
\text { mujeres sanos } \\
\text { (edad: } 32,7 \quad 7 \quad \pm \\
10.7 \text { ) }\end{array}$ & $\begin{array}{l}\text { No } \\
\text { especifica }\end{array}$ & Imagenología & $\begin{array}{l}\text { Observa un haz muscular anómalo entre el Músculo } \\
\text { Temporal (MT) y MPL. Se identifica en el 12,82\%, } \\
\text { distribuida por igual en ambos géneros. Se observan } \\
2 \text { patrones distintos de origen inserción. } \\
\text { Patrón A: Banda muscular con orientación } \\
\text { vertical, inserción en la superficie lateral del MPL y } \\
\text { fibras más mediales del MT. } \\
\text { Prevalencia de } 6,41 \% \text { en relación a las mues tras } \\
\text { totales. } \\
\text { Patrón B: Banda muscular con orientación } \\
\text { horizontal, inserción en la superficie lateral del } \\
\text { MPL y la superficie medial de las fibras anteriores } \\
\text { del MT. Prevalencia de } 6,41 \% \text { en relación a las } \\
\text { muestras totales. } \\
\text { Es considerado como el músculo "pterygoideus } \\
\text { proprius". }\end{array}$ \\
\hline
\end{tabular}

asociadas a las cabezas del MPL, mencionando de igual forma funciones similares asociadas a estas mismas, sin embargo, autores como Moore et al. (2010) se enfocan sólo a describir la función general del MPL sin hacer discriminación entre el MPL-S y MPL-I o Dauber \& Feneis (2007) y Antonopoulou et al. que asociarían funciones poco descritas del MPL-S, mencionando que este se encargaría de determinar la velocidad de arranque del DA y que sería activado durante la retrusión mandibular. Cada cabeza del MPL cumple funciones independientes, el MPL-S se caracterizaría por activarse durante la elevación de la mandíbula (Fun- ción más descrita) y actuar durante la masticación para apretar y mantener los dientes juntos, sería además un estabilizador del DA y CM durante la carga unilateral, en cambio el MPL-I tendría como función principal la protrusión, descenso de la mandíbula (Función más descrita) y actuando unilateralmente produciría los movimientos de lateralidad mandibular (Velayos \& Santana, 2001; Okeson, 2003; Biasotto; Nelson, 2015), la función de ambas cabezas seria empujar el DA, la CA y el CM hacia el tubérculo articular del hueso temporal produciendo el movimiento de protrusión mandibular (Velayos \& Santana, 1998). 
Tabla IV. Funciones del músculo pterigoideo lateral y sus cabezas.

\begin{tabular}{|c|c|c|}
\hline \multirow[t]{2}{*}{ Referencia } & \multicolumn{2}{|c|}{$\begin{array}{c}\text { Resultados } \\
\text { Músculo pterigoideo lateral }\end{array}$} \\
\hline & Cabeza superior & Cabeza inferior \\
\hline Biasotto, 2005 & $\begin{array}{l}\text { Actúa cuando hay resistencia muscular y } \\
\text { cuando los dientes se mantienen } \\
\text { cerrados durante la masticación. }\end{array}$ & $\begin{array}{l}\text { Al contra erse bilateralmente, los procesos } \\
\text { condilares se mueven hacia abajo en dirección al } \\
\text { tubérculo articular y se produce una protrusión. } \\
\text { Contracción unilateral, la mandíbula se mueve } \\
\text { lateralmente opuesta a la contracción. Presente en } \\
\text { movimiento de abertura. }\end{array}$ \\
\hline Nelson, 2015 & $\begin{array}{l}\text { Se activa en movimientos de cierre, } \\
\text { masticación y apretar los dientes durante } \\
\text { la deglución. Reubica el CM y DA } \\
\text { contra la eminencia articular durante el } \\
\text { movimiento de cierre mandibular. }\end{array}$ & $\begin{array}{l}\text { Colabora a la traslación del CM hacia abajo, } \\
\text { adelante y contralateralmente en la apertura de la } \\
\text { boca. }\end{array}$ \\
\hline $\begin{array}{l}\text { Velayos \& S antana, } \\
1998\end{array}$ & $\begin{array}{l}\text { Se activa durante los movimientos de } \\
\text { cierre de boca. Colabora en la } \\
\text { movilización del CM y del DA durante } \\
\text { el cierre. }\end{array}$ & $\begin{array}{l}\text { Toma acción durante los movimientos de abertura y } \\
\text { protrusión mandibular. }\end{array}$ \\
\hline Okeson, 2013 & $\begin{array}{l}\text { Cuando este músculo está contraído, las } \\
\text { fibras insertadas en el DA tiran de él } \\
\text { hacia adelante y hacia den tro. Actúa } \\
\text { como un protector del DA. } \\
\text { Se activa al morder con fuerza, al } \\
\text { mantener los dientes juntos y actuaría } \\
\text { junto a los músculos elevadores de la } \\
\text { mandíbula. }\end{array}$ & $\begin{array}{l}\text { Al contraerse bilateralmente, los CM son } \\
\text { traccionados desde las eminencias articulares hacia } \\
\text { abajo, y se produce una protrusión de la mandíbula. } \\
\text { La contracción unilateral crea un movimiento de } \\
\text { mediotrusión de ese cóndilo y origina un } \\
\text { movimiento lateral de la mandíbula hacia el lado } \\
\text { contrario. Cuando actúa con los depresores } \\
\text { mandibulares, la mandíbula desciende y los CM se } \\
\text { deslizan hacia adelante y hacia abajo sobre las } \\
\text { eminencias articulares. }\end{array}$ \\
\hline
\end{tabular}

Algunos autores han definido al MPL como dos músculos independientes (Juniper, 1981; Okeson, 2013), mientras que otros, afirman que esta hipótesis podría ser apoyada si se lograra identificar claramente la inervación separada de las cabezas del MPL (Aziz et al.). Un músculo con características similares es el esternocleidomastoideo, tiene una cabeza esternal y una clavicular, es inervado por el nervio accesorio (XI) y el plexo cervical (C1 - C2) (Dauber \& Feneis) y dentro de sus tantas funciones, puede realizar flexión cervical y extensión craneal. (Moore et al.). Con el fin de esclarecer estas teorías se analizó la literatura que evaluó la inervación del MPL, se concluyó la existencia de diferentes patrones nerviosos que se distribuían dentro del músculo, se identificaron nervios que se originaban tanto de una fuente en común (Aziz et al.) como de nervios independientes que podían inervar a ambas cabezas o a cada una de forma separada o adicional, todos estos surgieron desde el nervio mandibular (V3) proveniente del nervio trigémino (V) (Aziz et al.; Akita et al., 2000, 2001; Kim et al., 2003). Akita et al. (2000, 2001) identificaron la existencia de ramos proveniente del nervio temporal profundo anterior ingresando al MPL. En cambio, Kim et al. identificaron al tronco nervioso mandibular como la fuente que inerva al MPL. Aziz et al. además de identificar al nervio mandibular o bucal en el $75 \%$ de 20 cadáveres diseccionados, expone que en el $20 \%$ de las muestras la inervación fue de manera independiente para cada cabeza del MPL y esto fue dado por ramos del nervio temporal profundo anterior, bucal y/o mandibular. Ambas cabezas se les describió una inervación adicional, en el caso del MPL-S, esta provenía del nervio temporal profundo medio, bucal o mandibular y el MPL-I estos ramos provenían del nervio mandibular o bucal (Aziz et al.; Akita et al., 2000, 2001; Kim et al.). Estos ramos adicionales que podrían recibir tanto el MPL-S como el MPL-I es probable que puedan tener relación con los elementos recíprocos que inervaban al MPL descrito por Hiraba et al. (2000). Considerando lo anterior, si bien autores identifican que la inervación de ambas cabezas en algunos casos proviene de ramas nerviosas separadas e independientes, estas son la minoría, la mayoría de los autores concuerdan con que la inervación de ambas cabezas se origina de un nervio en común este puede ser el temporal profundo o mandibular, debido a esto nuestros resultados apoyan la teoría que las cabezas del MPL, no deben considerarse como músculos independientes. 
En cuanto al origen e inserción del MPL diferentes autores que evaluaron la ATM a través de disecciones en cadáveres japoneses, griegos y brasileros (rango edad: 40 y 79,6 años), identificaron elementos comunes de origen del MPL, entre estos destacaban la cresta infratemporal, el ala mayor y la lámina lateral del proceso pterigoides del hueso esfenoides (Siéssere et al., 2008; Matsunaga et al.; Antonopoulou et al.), si bien los autores generalmente están de acuerdo con el origen del MPL, existe algún grado de cuestionamiento en cuanto a su inserción, principalmente con la cabeza superior del MPL, ya que se podría unir solamente al DA de la ATM; solo al CM; al DA con pocas fibras en el CM o en el CM con pocas fibras en el DA (Carpentier et al.). Un estudio analizó la inserción del MPL$\mathrm{S}$ a través de la disección de 26 cadáveres e indicó que el 68 $\%$ de estos presentaba una inserción en la fóvea pterigoidea en el cuello del CM acompañado de una inserción secundaria, compuesta por una banda muscular que representaba el $20 \%$ del MPL-S, que se unía predominantemente a la CA. Otorgando además como información otra posibilidad de inserción del MPL-S, que sería en la CA (Wilkinson, 1988). En cambio, otros autores describirían que en este tipo inserción, la mayoría de las fibras, entre un $60 \%$ y $70 \%$, se insertaría en el cuello del CM y sólo entre un $30 \%$ a $40 \%$ se uniría al DA (Okeson, 2013). Haddioui et al., en un estudio el cual tuvo como objetivo evaluar 337 MPL en cadáveres, mediante técnicas de disección e imagenológicas, identificaron la existencia de una inserción en el DA a nivel de su extremidad medial y por otro lado en el cuello del CM de una forma triangular de base superior, finalmente concluye que el MPL debería considerarse como una unidad funcional, independiente de sus inserciones, ya que la escasa unión al DA asociado a la estructura peniforme del músculo contribuiría a los datos de funciones antagonistas de las cabeza superior e inferior registrados durante el ciclo masticatorio.

Según la evidencia actual, el origen del MPL no sería un tema controversial para los autores, pero si la inserción de este mismo, la cual no sería de la misma forma para todos los individuos, donde no existe consenso sobre el porcentaje de fibras que se insertan en las diferentes estructuras, pero sí coinciden en que la inserción del MPL-S más frecuente se daría en el cuello del CM, de una forma triangular, con pocas fibras en el DA en la superficie media de la cara anterior. Estas variaciones en los resultados podrían explicarse por las diferentes técnicas de disección o imagenológicas utilizadas y también por las características propias de los individuos observados, como ejemplo la variabilidad étnica.

Además de comprender los aspectos anatómicos y funcionales del MPL es importante en la práctica odontológica, conocer las posibles variaciones morfológicas que es propio de cada individuo (Snoeck et al., 2010). La mayoría de la literatura describe al MPL como un músculo compuesto por dos cabezas (El Haddioui et al.; Okeson, 2013). Sin embargo existen autores que mediante la utilización de técnicas, como disección e imagenología, han logrado identificar una tercera cabeza. Pompei Filho et al. (2009) al evaluar 178 ATMs a través de imágenes de resonancia magnética, identificaron una prevalencia de 20,22\% de una tercera cabeza del MPL, esta se insertó completamente en el DA, y podría estar posiblemente involucrada en el desarrollo de alteraciones y desplazamientos del DA. En cambio, Antonopoulou et al., al realizar la disección de 18 cadáveres a ambos lados, identificaron una tercera cabeza del MPL, a la cual designaron como "cabeza media del MPL" en el $22,2 \%$ de las muestras, pero debido a la posición de los orígenes del músculo y difícil la identificación de los márgenes del MPL, establecieron solo que el origen se daba en la superficie del ala mayor del hueso esfenoides. De una forma similar Akar et al. al diseccionar 50 cadáveres mencionaron la presencia de un origen del MPL a través de tres cabezas: superior, inferior y "cabeza interna".

A lo largo del tiempo se ha pensado que los músculos de la masticación están claramente delimitados pero recientes reportes indicarían la presencia de haces musculares entre estos mismos (Sakaguchi-Kuma et al., 2016). Akita et al. (2001), al diseccionar una muestra de 66 cadáveres observaron que en el 4,5\% existía una banda muscular que conectaba el músculo temporal (MT) y el MPL. Esto fue apoyado por Snoeck et al., quienes observaron 78 imágenes de resonancia magnética identificaron en el 12,82\% una banda muscular con características similares, identificando además dos tipos de orientaciones de esta banda (horizontal y vertical). Ambos autores describieron elementos de origen e inserción similares, esta se originaba de la superficie medial de las fibras anteriores o mediales del MT y se insertaba en la superficie ínfero - lateral del MPL, estos autores lo llamaron "pterygoideus proprius". Sin embargo, las funciones de estas variaciones anatómicas del MPL aún no están definidas y tampoco la incidencia que esto podría tener en el proceso masticatorio o en el funcionamiento de la ATM.

\section{CONCLUSIÓN}

El músculo pterigoideo lateral representa una estructura compleja y variable. Su origen se encuentra definido en la literatura sin mayores contradicciones, mientras que la inserción se ha estudiado en mayor proporción y aún sigue siendo tema de discusión en la literatura, especialmente la cabeza superior que puede tener múltiples inserciones. 
Las funciones del MPL generan mucha controversia, algunos autores han intentado describirlo como dos músculos independientes, sin embargo dentro los estudios analizados, la gran mayoría menciona que tienen un origen nervioso común, por lo que no es posible hablar de la división del músculo.

Consideramos que son insuficientes los estudios que analicen las funciones del MPL durante su acción, la mayoría de los estudios analiza cadáveres, por lo que creemos que el desarrollo de metodologías menos invasivas y dolorosas, ayudarían a comprender el comportamiento de este músculo durante su función y como las variaciones anatómicas influyen en éstas.

FARFÁN, C.; ROIG, J.; QUIDEL, B. \& FUENTES, R. Morphological and functional analysis of the lateral pterygoid muscle: A review of the literature. Int. J. Morphol., 38(6):17131721, 2020.

SUMMARY: The Lateral Pterygoid Muscle (LPM) is a complex and variable structure. Being able to determine its exact anatomy, neighboring relationships, origin and insertions, helps clinicians to better understand its function in the stomatognathic system. This review seeks to analyze the literature, in order to clarify the antagonistic function of the lateral pterygoid muscle heads, from a nervous point of view. Furthermore, the description of its origin, aspects of insertions and possible anatomical variations, its functions as reported in traditional literature, are analyzed and compared with original articles. Different electronic databases and books were analyzed, with designated inclusion and exclusion criteria. Two researchers independently reviewed the articles, whennecessary a third researcher resolved any differences. This review includes a total of 11 articles and 4 books related to our study topic. Results were reported using data extraction tables, which included functions, innervation, origin and insertion, and anatomical variations of the LPM. Our results show that the common elements of origin of LPM were clearly identified, but their insertion showed variations between the different studies, both in the percentage of fibers joined and the anatomical elements inserted. Considering the antagonistic functions of the two heads, authors have described it as two different muscles. However from a nervous point of view, this theory is not supported. Finally, understanding the functions of the LPM during its action is complex, since most studies available use cadavers or electromyography. Therefore, we believe that the development of less invasive and painful methodologies, would help to understand the influence of anatomical variations on the function of this muscle.

KEY WORDS: Lateral Pterygoid Muscle; Functional anatomy; Anatomical variations; Temporomandibular joint.

\section{REFERENCIAS BIBLIOGRÁFICAS}

Akar, G. C.; Govsa, F. \& Ozgur, Z. Examination of the heads of the lateral pterygoid muscle on the temporomandibular joint. $J$. Craniofac. Surg., 20(1):219-23, 2009.

Akita, K.; Shimokawa, T. \& Sato, T. Aberrant muscle between the temporalis and the lateral pterygoid muscles: M. pterygoideus proprius (Henle). Clin. Anat., 14(4):288-91, 2001.

Akita, K.; Shimokawa, T. \& Sato, T. Positional relationships between the masticatory muscles and their innervating nerves with special reference to the lateral pterygoid and the midmedial and discotemporal muscle bundles of temporalis. J. Anat., 197(Pt. 2):291$302,2000$.

Alves, N. \& Candido, P. L. Anatomía Aplicada a la Odontología. São Paulo, Santos, 2009.

Antonopoulou, M.; Iatrou, I.; Paraschos, A. \& Anagnostopoulou, S. Variations of the attachment of the superior head of human lateral pterygoid muscle. J. Craciomaxillofac. Surg., 41(6):e91-7, 2013.

Aziz, M. A.; Cowie, R. J.; Skinner, C. E.; Abdi, T. S. \& Orzame, G. Are the two heads of the human lateral pterygoid separate muscles? A perspective based on their nerve supply. J. Orofac. Pain, 12(3):22639, 1998.

Bernal-Mañas, C. M.; González-Sequeros, O.; Moreno-Cascales, M.; Sarria-Cabrera, R. \& Latorre-Reviriego, R. M. New anatomoradiological findings of the lateral pterygoid muscle. Surg. Radiol. Anat., 38(9):1033-43, 2016.

Biasotto, D. A. Abordagem Interdisciplinar das Disfuncoes Temporomandibulares. São Paulo, Manole, 2005.

Carpentier, P.; Yung, J. P.; Marguelles-Bonnet, R. \& Meunissier, M. Insertions of the lateral pterygoid muscle: an anatomic study of the human temporomandibular joint. J. Oral Maxillofac. Surg., 46(6):477-82, 1988.

Dauber, W. \& Feneis, H. Feneis. Nomenclatura Anatómica Ilustrada. $5^{\mathrm{a}}$ ed. Madrid, Elsevier Masson, 2007.

El Haddioui, A.; Laison, F.; Zouaoui, A.; Bravetti, P. \& Gaudy, J. Functional anatomy of the human lateral pterygoid muscle. Surg. Radiol. Anat., 27(4):271-86, 2005.

Fernández, L. I.; Zanotta, G. \& Kreiner, M. Estudio comparativo del complejo electromiográfico post-estímulo del músculo masetero en pacientes rehabilitados con prótesis completa bimaxilar mediante técnica piezográfica y técnica convencional. Odontoestomatología, 12(14):45-53, 2010.

Ferrario, V. \& Sforza, C. Biomechanical model of the human mandible: a hypothesis involving stabilizing activity of the superior belly of lateral pterygoid muscle. J. Prosthet. Dent., 68(5):829-35, 1992.

Gutiérrez, L. \& Grossman, E. Anatomofisiologia do músculo pterigóideo lateral. Rev. Dor, 11(3):249-53, 2010.

Hiraba, K.; Hibino, K.; Hiranuma, K. \& Negoro, T. EMG activities of two heads of the human lateral pterygoid muscle in relation to mandibular condyle movement and biting force. J. Neurophysiol., 83(4):2120-37, 2000.

Juniper, R. The superior pterygoid muscle? Br. J. Oral Surg., 19(2):1218, 1981.

Kendall, F.; McCreary, E.; Provance, P.; Rodgers, M. \& Romani, W. Kendall's. Músculos, Pruebas Funcionales, Postura y Dolor. Madrid, Marbán, 2005.

Kim, H. J.; Kwak, H. H.; Hu, K. S.; Park, H. D.; Kang, H. C.; Jung, H. S. $\&$ Koh, K. S. Topographic anatomy of the mandibular nerve branches distributed on the two heads of the lateral pterygoid. Int. J. Oral Maxillofac. Surg., 32(4):408-13, 2003.

Langton, D. P. \& Eggleton, T. M. Functional Anatomy of the Temporomandibular Joint Complex. Santiago de Chile, IFORC, 1992.

Lopes, A. Anatomia Cabeca e Pescoco. Rio de Janeiro, Guanabara Koogan, 2004. 
Matsunaga, K.; Usui, A.; Yamaguchi, K. \& Akita, K. An anatomical study of the muscles that attach to the articular disc of the temporomandibular joint. Clin. Anat., 22(8):932-40, 2009.

Mazza, D.; Marini, M.; Impara, L.; Cassetta, M.; Scarpato, P.; Barchetti, F. $\&$ Di Paolo, C. Anatomic examination of the upper head of the lateral pterygoid muscle using magnetic resonance imaging and clinical data. J. Craniofac. Surg., 20(5):1508-11, 2009.

Moore, K.; Dalley, A. \& Agur, A. Anatomía con Orientación Clínica. 6a ed. Barcelona, Lippincott Williams \& Wilkins, 2010.

Naidoo, L. C. D. \& Juniper, R. P. Morphometric analysis of the insertion of the upper head of the lateral pterygoid muscle. Oral Surg. Oral Med. Oral Pathol. Oral Radiol. Endod., 83(4):441-6, 1997.

Nelson, S. J. Anatomia. Fisiología y Oclusión Dental. Barcelona, Elsevier Masson, 2015.

Norton, N. S. Netter. Anatomía de Cabeza y Cuello para Odontólogos. Barcelona, Elsevier Masson, 2007.

Okeson, J. P. Tratamiento de Oclusión y Afecciones Temporomandibulares. $5^{\text {a }}$ ed. Madrid, Elsevier Masson, 2003.

Okeson, J. P. Tratamiento de Oclusión y Afecciones Temporomandibulares. $7^{\mathrm{a}}$ ed. Barcelona, Elsevier Inc, 2013.

Pompei Filho, H.; Guimaraes, A. S. \& Suazo Galdames, I. Prevalence of the third head of the lateral pterygoid muscle: a magnetic resonance image study. Int. J. Morphol., 27(4):1043-6, 2009.

Pompei Filho, H.; Suazo Galdames, I. \& Guimaraes, A. S. Superior head of the lateral pterygoid muscle inserting in asymptomatic temporomandibular joints. Int. J. Odontostomat., 4(1):19-22, 2010.

Sakaguchi-Kuma, T.; Hayashi, N.; Fujishiro, H.; Yamaguchi, K.; Shimazaki, K.; Ono, T. \& Akita, K. An anatomic study of the attachments on the condylar process of the mandible: muscle bundles from the temporalis. Surg. Radiol. Anat., 38(4):461-7, 2016.

Siéssere, S.; Vitti, M.; Semprini, M.; Regalo, S. C. H.; Iyomasa, M. M.; Dias, F. J.; Issa, J. P. M. \& de Sousa, L. G. Macroscopic and microscopic aspects of the temporomandibular joint related to its clinical implication. Micron, 39(7):852-8, 2008.

Snoeck, T.; Provyn, S.; Balestra, C.; Parlak, B.; Emonts, P.; Sesbouë, B. \& Clarys, J. P. The musculus pterygoïdeus proprius: an in-vivo approach with magnetic resonance imaging. J. Anat., 217(6):679-82, 2010.

Testut, L. \& Latarjet, A. Compendio de Anatomía Descriptiva. Barcelona, Elsevier Masson, 1989.

Usui, A.; Akita, K. \& Yamaguchi, K. An anatomic study of the divisions of the lateral pterygoid muscle based on the findings of the origins and insertions. Surg. Radiol. Anat., 30(4):327-33, 2008.

Velayos J. L. \& Santana H. Anatomía de la Cabeza Con Enfoque Odontoestomatológico. $3^{\mathrm{a}}$ ed. Madrid, Médica Panamericana, 2001.

Velayos, J. L. \& Santana H. Anatomía de la Cabeza con Enfoque Odontoestomatológico. $2^{\mathrm{a}}$ ed. Madrid, Médica Panamericana, 1998.

Wildmalm, S. E.; Lillie, J. H. \& Ash Jr., M. M. Anatomical and electromyographic studies of the lateral pterygoid muscle. J. Oral Rehabil., 14(5):429-46, 1987.

Wilkinson, T. M. The relationship between the disk and the lateral pterygoid muscle in the human temporomandibular joint. J. Prosthet. Dent., 60(6):715-24, 1988.
Corresponding Author

Prof. Dr. Ramón Fuentes Fernández

Research Centre for Dental Sciences

Dental School

Universidad de La Frontera

Av. Francisco Salazar 1145

Temuco

CHILE

Email: ramon.fuentes@ufrontera.cl

Received: 22-04-2020

Accepted: 23-07-2020 